\title{
Static and Dynamic Structural Response of an Aircraft Wing with Damage Using Equivalent Plate Analysis
}

\author{
T. Krishnamurthy* \\ NASA Langley Research Center, Hampton, VA 23681, U.S.A. \\ and \\ Frank J. Tsai \\ Lockheed Martin Mission Services, Hampton, VA 23681, U.S.A.
}

\begin{abstract}
A process to generate an equivalent plate based on an optimization approach to predict the static and dynamic response of flight vehicle wing structures is proposed. Geometricscale and frequency-scale factors are defined to construct an equivalent plate with any desired scale to use in simulation and wind tunnel experiments. It is shown that the stiffness and the displacements are scaled linearly with the geometric-scale factor, whereas the load is scaled as the square of the geometric-scale factor. The scaled stiffness of the reference flight vehicle is matched first to construct the equivalent plate. Then the frequency-scale factor is defined to scale the flight vehicle frequencies. The scaled flight vehicle frequencies are matched by placing arbitrary point masses along the equivalent plate geometry. Two simple stiffened-plate examples, one with damage and another without damage, were used to demonstrate the accuracy of the optimization procedure proposed. Geometric-scale factors ranging from 0.2 to 1.0 were used in the analyses. In both examples, the static and dynamic response of the reference stiffened-panel solution is matched accurately. The scaled equivalent plate predicted the first five frequencies of the stiffened panel very accurately. Finally, the proposed equivalent plate procedure was demonstrated in a more realistic typical aircraft wing structure. Two scale equivalent plate models were generated using the geometric-scale factors 1.0 and 0.2 . Both equivalent plate models predicted the static response of the wing structure accurately. The equivalent plate models reproduced the first five frequencies of the wing structure accurately.
\end{abstract}

\section{Introduction}

Understanding the effects of discrete source damage (e.g. uncontained rotor burst) on the response of aircraft structures is necessary to improve the survivability of future aircraft to adverse damage events. Rapid modeling and analysis methods are also key requirements to generate static and dynamic load constraints for an adaptive control system and for vehicle health management in the presence of structural damage. To generate damage-dependent structural constraints for an adaptive control system and to verify that the control frequency is sufficiently separate from the structural frequencies, it is necessary to determine the frequency response of the structures in the presence of damage. Frequency estimation is also necessary to determine the flutter boundary changes due to the damage. Hence, there is a need to develop methods to determine the frequency response of the aircraft wing in the presence of damage. One such method is equivalent plate analysis.

Equivalent plate analysis is often used to replace the computationally expensive detailed finite element analysis in initial design stages or in conceptual design of aircraft wing structures [1]. In equivalent plate modeling, the model characteristics are represented with polynomials, which require only a small fraction of the input data that would be required by a corresponding finite element model. The equivalent plate model can also be used to design a wind tunnel model to match the stiffness characteristics of the wing box of a full-scale flight vehicle model while

\footnotetext{
* Aerospace Engineer, Durability, Damage Tolerance and Reliability Branch, Associate Fellow, AIAA
} 
satisfying strength-based requirements [2]. An equivalent plate analysis procedure based on the Ritz method was proposed at NASA Langley Research Center as early as 1986 [3]. In the Ritz method-based equivalent plate theory, the aircraft wing structure is modeled with several trapezoidal segments. Several modifications and improvements to the initial theory proposed in reference 3 resulted in the development of a structural analysis code ELAPS (Equivalent Laminated Plate Solution) [4, 5]. The ELAPS code solutions predict the displacement, stress, and mode shape calculations within five to ten percent of a comparable finite element solution [6]. However, the Ritz methodbased equivalent plate theory in the ELAPS code is not easily amenable to implement in general-purpose commercial structural finite element analysis codes.

Recently, in reference 7, an equivalent plate procedure was developed to provide a computationally efficient means of matching the stiffness and frequencies of flight vehicle wing structures for prescribed loading conditions. First, the equivalent plate is used to match the stiffness of a stiffened panel without damage and the stiffness of a stiffened panel with damage. For both stiffened panels, the equivalent plate models reproduce the deformation of a corresponding detailed model accurately for the given loading conditions. Once the stiffness was matched, the equivalent plate models were then used to predict the frequencies of the panels. Two analytical procedures using the lumped-mass matrix were used to match the first five frequencies of the corresponding detailed model. In both the procedures, the lumped-mass matrix for the equivalent plate is constructed by multiplying the diagonal terms of the consistent-mass matrix by a proportionality constant. The authors found that considering only metallic materials for the equivalent plate (and thus varying density alone) did not provide enough flexibility to match all the desired frequencies. Large errors were noted, especially at higher frequencies [7]. Hence it was decided to extend the work by considering composite and sandwich construction for equivalent plate. In reference 8, two approaches were presented to match the frequency response of the wing structures using an equivalent plate model. In the first approach, the equivalent plate was generated by only matching the stiffness of the flight vehicle wing structure and then the frequencies were matched by adding point masses at discrete locations along the equivalent plate. The second approach generated the equivalent plate by matching both the stiffness and the frequencies of the flight vehicle wing structure simultaneously. Several candidates for equivalent plates were considered including composite material, foam core sandwiches and multizone metallic materials. Even though both stiffness and frequencies are matched accurately, the authors felt that the fabrication of the equivalent plate generated will continue to pose a challenge. Hence, there is a need to develop similarity factors to suitably scale down the equivalent plate so it can be easily fabricated. Also, there is a need to develop similarity scale factors to construct scaled equivalent plates that can be used in simulation and wind tunnel models. Additionally, there is a need to demonstrate the equivalent plate procedures developed in references 7 and 8 are applicable to more realistic aircraft structures.

In this paper, similarity scaling factors to scale down the equivalent plate to predict the static and dynamic response of an aircraft wing structure are developed. A geometric scale factor is proposed to predict the static response of the aircraft wing structures. Also, a frequency-scale factor is proposed to predict the dynamic response. The analysis techniques previously developed in references 7 and 8 are extended and applied to generate scaled down equivalent plates for an aircraft wing model to use in simulation and wind tunnel experiments.

The paper is constructed as follows. First, a geometric scale factor along with an optimization procedure is defined to predict the static response of the aircraft wing structures. Second, a frequency scale factor is defined with an optimization procedure to predict the dynamic response of the flight vehicle wing structures. Third, two simple numerical examples, one without damage and another with damage, are presented to validate the scaling factors selection as well as the equivalent plate generation procedures. Fourth, the equivalent plate procedure and the scale factors selection are demonstrated in a realistic, typical aircraft wing structure. Finally, a brief summary is presented. 


\section{Geometric-Scale Factor and Scaled Stiffness Matching}

It is often necessary to construct a scaled-down equivalent plate model to predict the static response of flight wing structures in simulation and in wind tunnel experiments. To simulate the lift, drag, and moment characteristics of a flight vehicle wing structure using an equivalent plate, it is sufficient to simulate the static load-deflection of the wing structure [9]. The stiffness of the equivalent plate and the flight vehicle wing-structure are similar when the nondimensional lift distribution $C_{p}(x, y)$ produces the same nondimensional deflection [9]

$$
\frac{w_{m}}{\sqrt{A_{m}}}=\frac{w_{f}}{\sqrt{A_{f}}}
$$

$$
\begin{aligned}
& \text { Where, } \\
& w_{m}-\text { is the scaled deflection of the equivalent plate } \\
& w_{f}-\text { is the actual deflection of the flight vehicle } \\
& A_{m}-\text { is the scaled wing area of the equivalent plate } \\
& A_{f}-\text { is the actual wing area of the flight vehicle }
\end{aligned}
$$

The subscripts $m$ and $f$ in Eq. (1) represent the scaled equivalent plate model and the flight wing structure, respectively.

At this point, a geometric scale factor $S_{g}$ is selected such that

$$
\begin{aligned}
& S_{g}=\sqrt{\frac{A_{m}}{A_{f}}} \\
& \frac{\left(\Delta A_{m}\right)_{i}}{A_{m}}=\frac{\left(\Delta A_{f}\right)_{i}}{A_{f}}
\end{aligned}
$$

where $\left(\Delta A_{m}\right)_{i}$ and $\left(\Delta A_{f}\right)_{i}$ are the corresponding elemental areas located at a given panel point $i$. The equations (2) and (3) implies that the equivalent plate is uniformly scaled in both length and width direction of the flight vehicle wing geometry.

The aerodynamic load at any elemental area $\Delta A_{i}$ located at a given panel point $i$ is:

$$
P_{i}=q C_{P_{i}} \frac{\Delta A_{i}}{A} A
$$

This load at point $i$ produces a deflection $w_{j}$ at an arbitrary point $j$. The load-deflection relationship in terms of the stiffness $K_{i j}$ can be written as

$$
P_{i}=K_{i j} w_{j}
$$


The nondimensional deflection in Eq. (1) can be rewritten using Eqs. (4) and (5) as

$$
\frac{w_{i}}{\sqrt{A}}=q C_{P_{i}} \frac{1}{K_{i j}} \frac{\Delta A_{i}}{A} \frac{A}{\sqrt{A}}
$$

In representing Eq. (6), the subscripts $i$ and $j$ are dropped. Neglecting the effect of Reynolds number on $C_{P_{i}}$, there will be aerodynamic flow similarity (equal $C_{P_{i}}$ ) between the flight vehicle wing-structure and the equivalent plate model, if the model is tested at the same Mach number [9]. Using Eqs. (3) and (6) in Eq. (1), the required similarity requirement for the stiffness between the equivalent plate model and the flight vehicle can be written as:

$$
\frac{q_{m}}{q_{f}} \frac{K_{f}}{K_{m}}\left(\frac{A_{m}}{A_{f}}\right)^{\frac{1}{2}}=1
$$

For every choice of Mach number and altitude of the airplane, there will be a corresponding Mach number and dynamic pressure can be set in the model [10]. In the present study, the dynamic pressure scale is selected such that the dynamic pressure in the equivalent plate and the flight vehicle are assumed to be the same, i.e. $q_{m}=q_{f}$.

Substituting Eq. (2) in Eq. (7), the scaled stiffness for the equivalent plate can be expressed in terms of the flight vehicle stiffness as

$$
K_{m}=S_{g} K_{f}
$$

Eq. (8) implies that the stiffness of the equivalent plate is scaled the same as the geometric scaling.

The relationship between the deformation of the scaled equivalent plate and the deformation of the flight vehicle can be written using Eqs. (1) and (2) as

$$
w_{m}=S_{g} w_{f}
$$

Similarly the relationship between the applied load in the equivalent plate and the applied load in the flight structure can be written using Eqs. (5) and (8) as

$$
P_{m}=S_{g}^{2} P_{f}
$$

Eqs. (8) to (10) completely define the quantities needed for equivalent plate construction. Once the geometric-scale factor $S_{g}$ is selected, the load, stiffness and deformation of the scaled equivalent plate can be determined using Eqs. (8) to (10). In summary, once the geometric-scale factor is fixed,

1. Stiffness is scaled by the geometric-scale factor (Eq. 8)

2. Deformation is also scaled by the geometric-scale factor (Eq. 9)

3. Load is scaled by the square of the geometric-scale factor Eq. (10)

Once the load, displacement and stiffness ratios are determined (using Eqs. 8 to 10), the following procedure adopted from references 7 and 8 is used to construct the equivalent plate by finding the optimum thickness distribution of the plate: 
1. Define or select the geometric scaling $S_{g}$.

2. Obtain the displacement field of the flight vehicle by performing a full scale finite element linear static analysis for the given loading and boundary conditions.

3. Using the geometric scaling, fix the dimensions of the equivalent plate planform.

4. Construct the finite element model of the equivalent plate.

5. If the nodal locations of the equivalent plate model are different from that of the full-scale fight vehicle finite element nodes, interpolate displacements at the equivalent plate nodal locations. In the present study, the nodes in the equivalent plate are located at the same location as the reference flight vehicle nodes, hence no interpolation was used.

6. The applied load for the equivalent plate is obtained using $P_{m}=S_{g}^{2} P_{f}$

7. Find the displacement distribution of the equivalent plate by performing linear static analysis.

8. Optimum thickness distribution of the equivalent plate is determined by minimizing the sum of the differences between the displacements of the flight vehicle and the equivalent plate model.

The objective function $\Phi$ for the optimization problem can be stated as

$$
\Phi=\sum_{i=1}^{N}\left(w_{m}{ }^{i}-S_{g} w_{f}{ }^{i}\right)^{2}
$$

where,

$N$ - is the number of finite element nodes in the equivalent plate model;

$w_{m}^{i}-$ is the displacement at the $i^{t h}$ node of the equivalent plate; and

$w_{f}^{i}-$ is the interpolated displacement of the flight vehicle at the location

of the $i^{\text {th }}$ node of the equivalent plate.

The objective function $\Phi$ in Eq. (11) is minimized in the optimization procedure to obtain the thickness distribution of the equivalent plate subject to the load constraint

$$
P_{m}=S_{g}^{2} P_{f}
$$

The optimized thickness distribution of the equivalent plate will produce the reduced stiffness of the scaled equivalent plate as

$$
K_{m}=S_{g} K_{f}
$$

In the present study, the general purpose finite element code ABAQUS ${ }^{\circledR}[11]^{\dagger}$ is used for the finite element analysis and the optimization code $\mathrm{DOT}^{\circledR}[12]$ is used in the optimization procedure.

\footnotetext{
${ }^{\dagger}$ Trade names and trademarks are used in this report for identification only. Their usage does not constitute an official endorsement, either expressed or implied, by the National Aeronautics and Space Administration.
} 


\section{Frequency-Scale Factor and Frequency Matching}

In the present paper, the frequency response of the equivalent plate is matched after matching the scaled stiffness of the equivalent plate as described in the previous section. Since matching the scaled stiffness fixes the geometry, material, and mass of the equivalent plate, it is very difficult to match the frequencies of the flight vehicle due to the relatively large mass of the equivalent plate. In this paper, a procedure to match the frequency response of the equivalent plate to the scaled frequencies of the flight vehicle is described. For this purpose, an independently selected frequency-scale factor is defined to link the frequency of the equivalent plate to the flight vehicle frequency as

$$
\omega_{m}=S_{\omega} \omega_{f}
$$

Where,

$$
\begin{aligned}
& S_{\omega}-\text { Frequency-scale factor to match the flight vehicle } \\
& \omega_{m}-\text { Frequency of the scaled equivalent plate } \\
& \omega_{f}-\text { Frequency of the reference flight vehicle wing structure }
\end{aligned}
$$

Using the scale factor, the first fundamental natural frequency of the flight vehicle and equivalent plate model is written as

$$
\omega_{m}^{1}=S_{\omega} \omega_{f}^{1}
$$

or

$$
\sqrt{\frac{K_{m}}{M_{m}}}=S_{\omega} \sqrt{\frac{K_{f}}{M_{f}}}
$$

or

$$
S_{\omega}=\sqrt{S_{g} \frac{M_{f}}{M_{m}}}
$$

where $M_{m}$ and $M_{f}$ represents the total mass of the equivalent plate and flight vehicle wing structure, respectively.

In general, the frequency-scale factor calculated using Eq. (15) will not match even the first frequency. In order to match a range of frequencies (for example, the first five), Eq. (15) can be modified and written as

$$
S_{\omega}=\sqrt{S_{g} \frac{M_{f}}{\left(M_{m}+\varepsilon_{m}\right)}}
$$


where $\varepsilon_{m}$ is total mass placed at arbitrary number of points as concentrated masses placed along the length and width of the equivalent plate to match the range of frequencies (for example, the first five). At this point, the locations and values of the concentrated masses are unknown. To determine the frequency-scale factor and the number, locations, and the values of the concentrated masses, the following optimization procedure is used in the present study:

1. Pre-select the number of concentrated masses to be placed, say $N_{\varepsilon}$

2. Select the number of frequencies to be matched, say 5

3. Then the value of the frequency scale-scale factor and values of the concentrated masses are determined by minimizing the objective function

$$
\psi=\sum_{i=1}^{N_{\varepsilon}} \xi_{m}^{i}
$$

where,

$$
\begin{aligned}
& \xi_{m}^{i} \text { - are the concentrated masses placed at pre-determined places in the model } \\
& \qquad\left(i=1,2,3, \cdots N_{\varepsilon}\right)
\end{aligned}
$$

The minimization problem in Eq. (17) is solved subject to the constraint

$$
\begin{gathered}
G(i)=\omega_{m}^{i}-S_{\omega} \omega_{f}^{i} \leq \text { Tolerence } \\
(\text { for } i=1,2, \cdots 5)
\end{gathered}
$$

The values of the concentrated masses $\xi_{m}^{i}$ and the frequency-scale factor are unknowns in the optimization. The tolerance can be pre-selected in the optimization procedure.

4. At the end of optimization procedure, the value of the frequency-scale factor is determined such the total concentrated masses to be added to the equivalent plate and errors in the frequencies are minimized.

\section{Numerical Example: Simple Structures}

The equivalent plate procedure is demonstrated on a set of simple structures that simulate a cantilevered wing. In the first model, a stiffened panel with two blade stiffeners without damage is considered. In the second model, the stiffened panel is modified to include discrete source damage in the form of a circular hole in the center of the panel. Three different geometric scale factors $S_{g}=1.0,0.5,0.2$ were selected. In the first step, the equivalent plate geometries are determined by matching the stiffness of the reference model for a given geometric-scale factor. In the second step, the frequency-scale factor and values of concentrated masses to be added to the equivalent plate are estimated to match the frequency response of the reference model. The results obtained from these analyses are discussed for the two models. 


\section{Model 1: Stiffened-Panel without Damage:}

The two-blade stiffened panel used to generate the equivalent plate model is shown in Figure 1. The dimensions, material properties and the loading used in the analysis are also shown in the figure.

The plate is completely fixed (cantilevered) at the edge CD. Two types of loading are applied simultaneously at the free edge $A B$. The first is a uniform vertical displacement $-w_{B}$ that is applied at the free edge $A B$ to simulate a bending loading. The second is a torsional loading that is simulated by linearly varying the vertical displacement from $+w_{T}$ at point $\mathrm{A}$ to $-w_{T}$ at point $\mathrm{B}$.

The finite element model of the stiffened panel and its equivalent plate model are shown in Figures 2 and 3, respectively. The nodes in the equivalent plate exactly match the nodes in the stiffened panel, thus eliminating the need for displacement interpolation in step 5 of the optimization procedure. Since the bending and torsional loads are simulated by applying displacement boundary conditions at the free edge, the total load applied is obtained by summing the reactions at the fixed edge in each of the models.
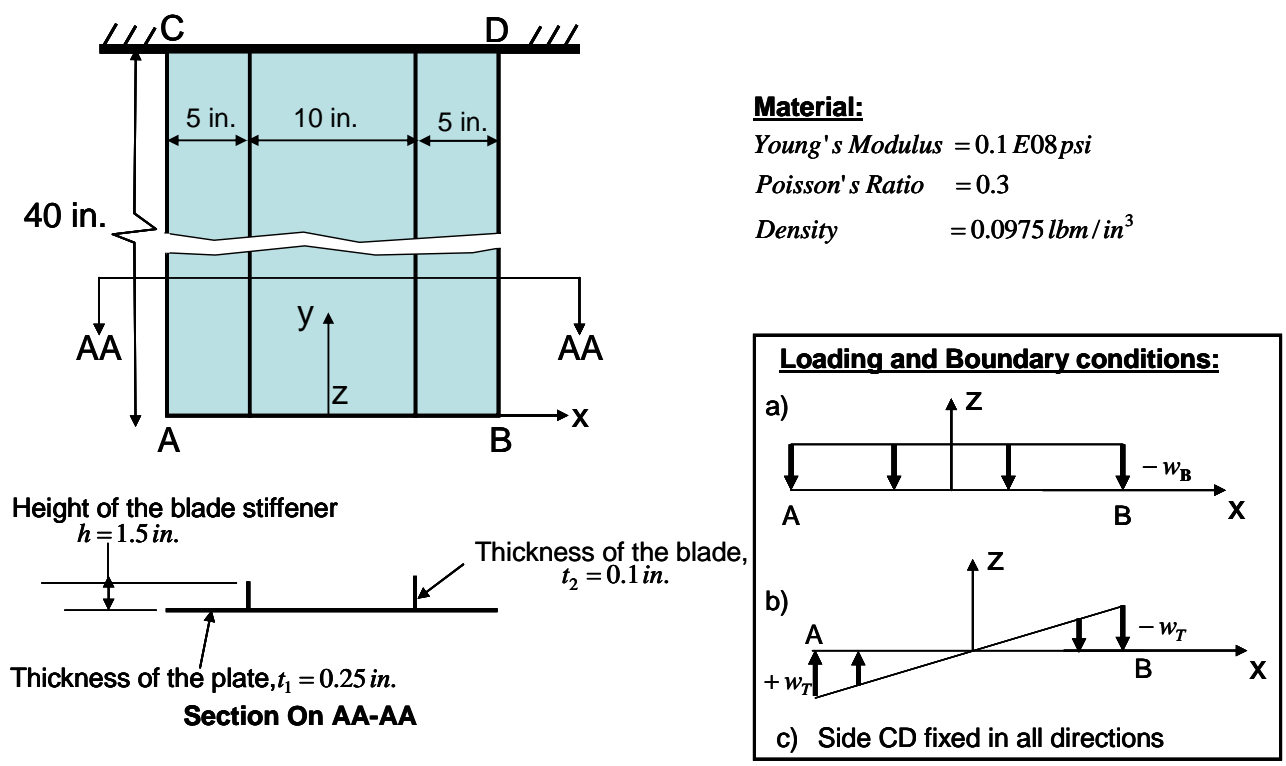

Figure 1. Geometry of the stiffened panel used in the equivalent plate generation

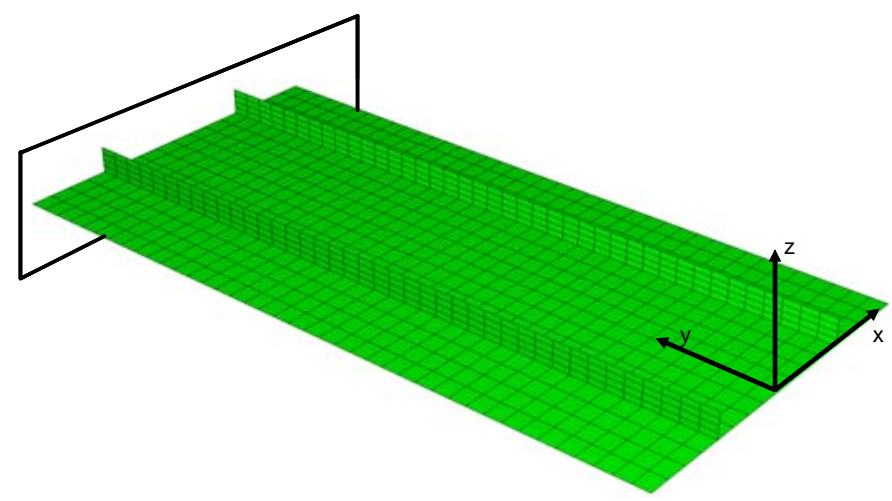


Figure 2. Finite element model of the stiffened panel reference model

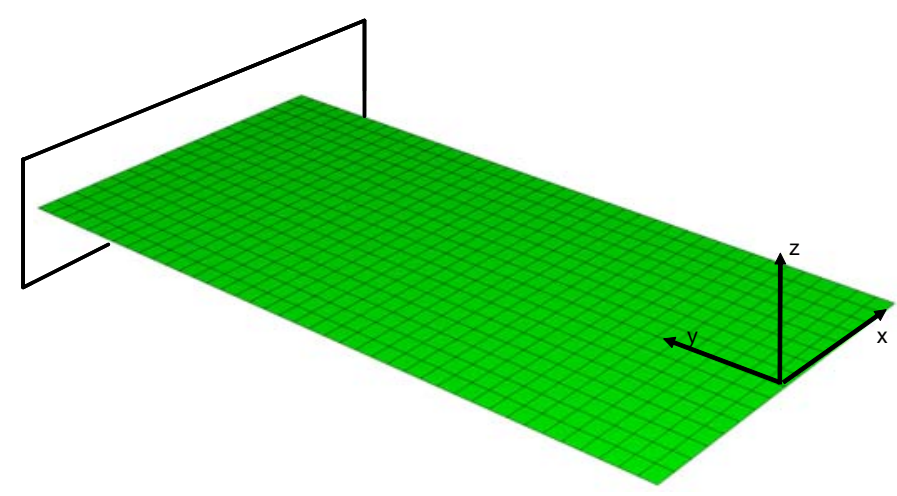

Figure 3. Finite element model of the equivalent plate

Linear variation of the thickness is assumed along the length of the plate. The thickness distribution obtained for scale factor 1.0 is presented in reference 8. Similar results proportional to the geometrical scaling were obtained for scale factor 0.5 and 0.2 and are not reported here. Results for the stiffness matching for the geometric-scale factor 1.0 is also already presented in reference 8 , so the deformation comparisons are shown only for geometric-scale factor 0.5 and 0.2 in Figure 4.

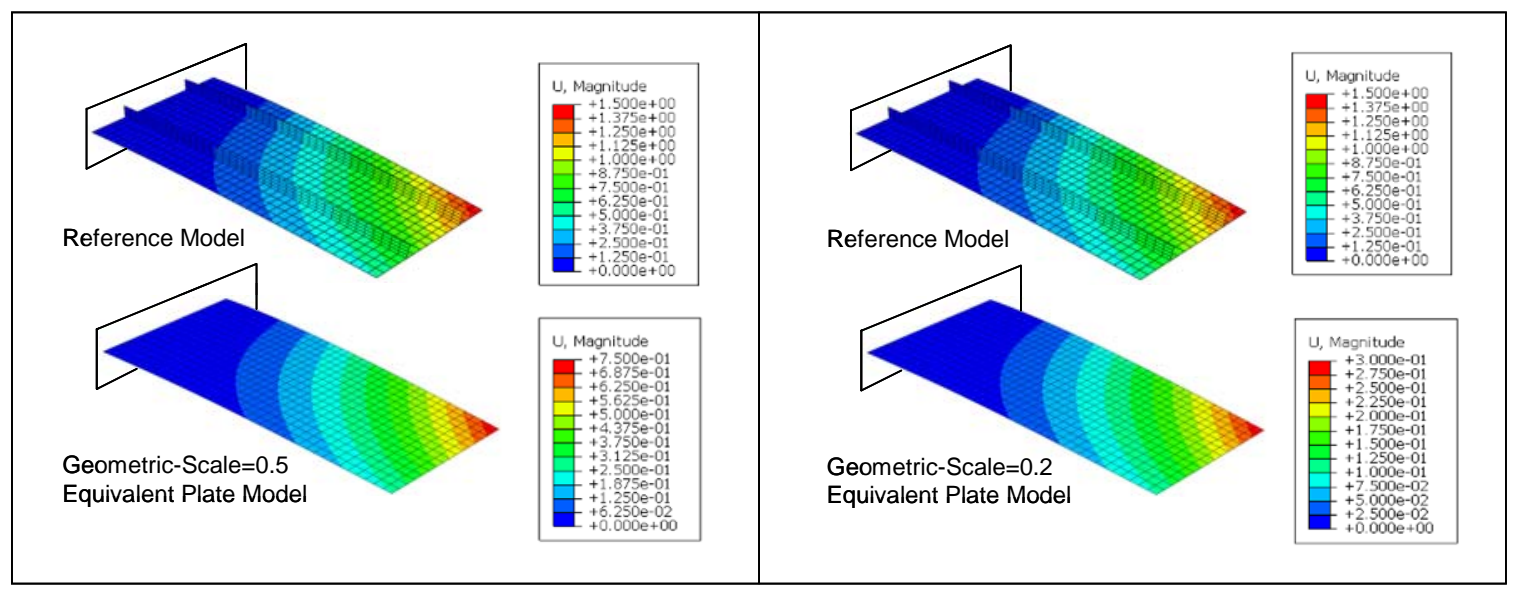

Figure 4. Stiffened panel without damage: deformation comparison for geometric scale factors 0.5 and 0.2

The geometric scale factors and frequency comparisons for the stiffened-plate without damage is shown in Figure 5, along with total mass added in each case. The maximum error in the frequencies is less than 2 percent in all cases. 

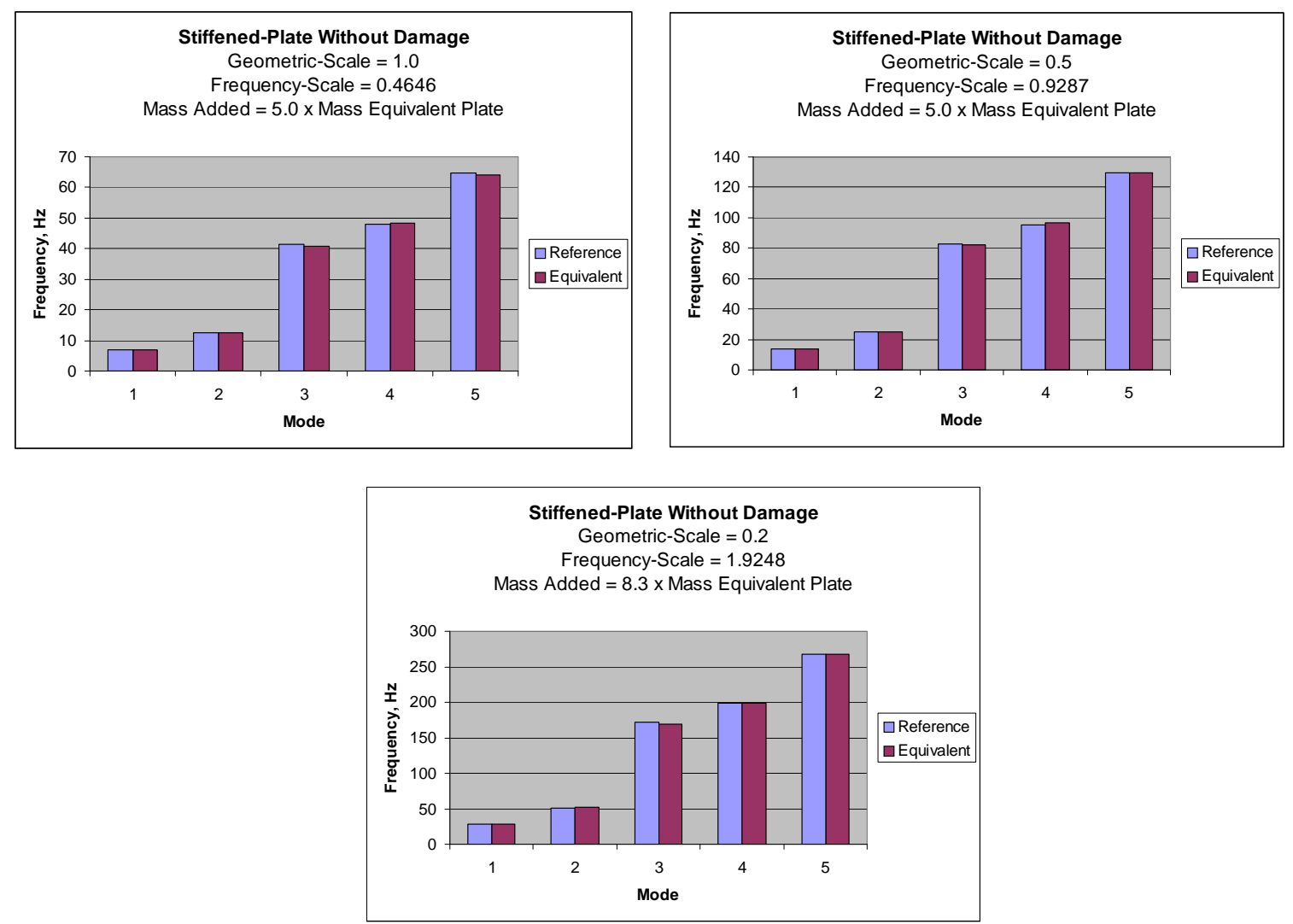

Figure 5. Stiffened-panel without damage: Frequency matching for geometric-scale factors 1.0, 0.5 and 0.2

The various frequency-scale factors and mass added for various geometric-scale factors are shown in Table 1.

Table 1.Stiffened-panel without damage: Frequency-scale factors and mass added

\begin{tabular}{|c|c|c|}
\hline Geometric-Scale & Frequency-Scale & Mass Added \\
\hline 1.0 & 0.4646 & $5.0 \times$ Mass Equivalent Plate \\
\hline 0.5 & 0.9287 & $5.0 \times$ Mass Equivalent Plate \\
\hline 0.2 & 1.9248 & $8.3 \times$ Mass Equivalent Plate \\
\hline
\end{tabular}

It can be concluded from the table that the total concentrated additional mass required varies from 5 to 8.3 times the mass of the equivalent plate. These values of the masses are obtained by placing 10 point masses in the length direction and 2 point masses in the width direction. No attempt is made to optimize the location of theses masses to reduce the total mass added to the plate.

The mode shapes for all the geometric-scale factors look almost the same; the mode shapes are compared only for the geometric-scale factor of 0.5 in Figure 6. Lower mode shapes agree better than the higher mode shapes. 


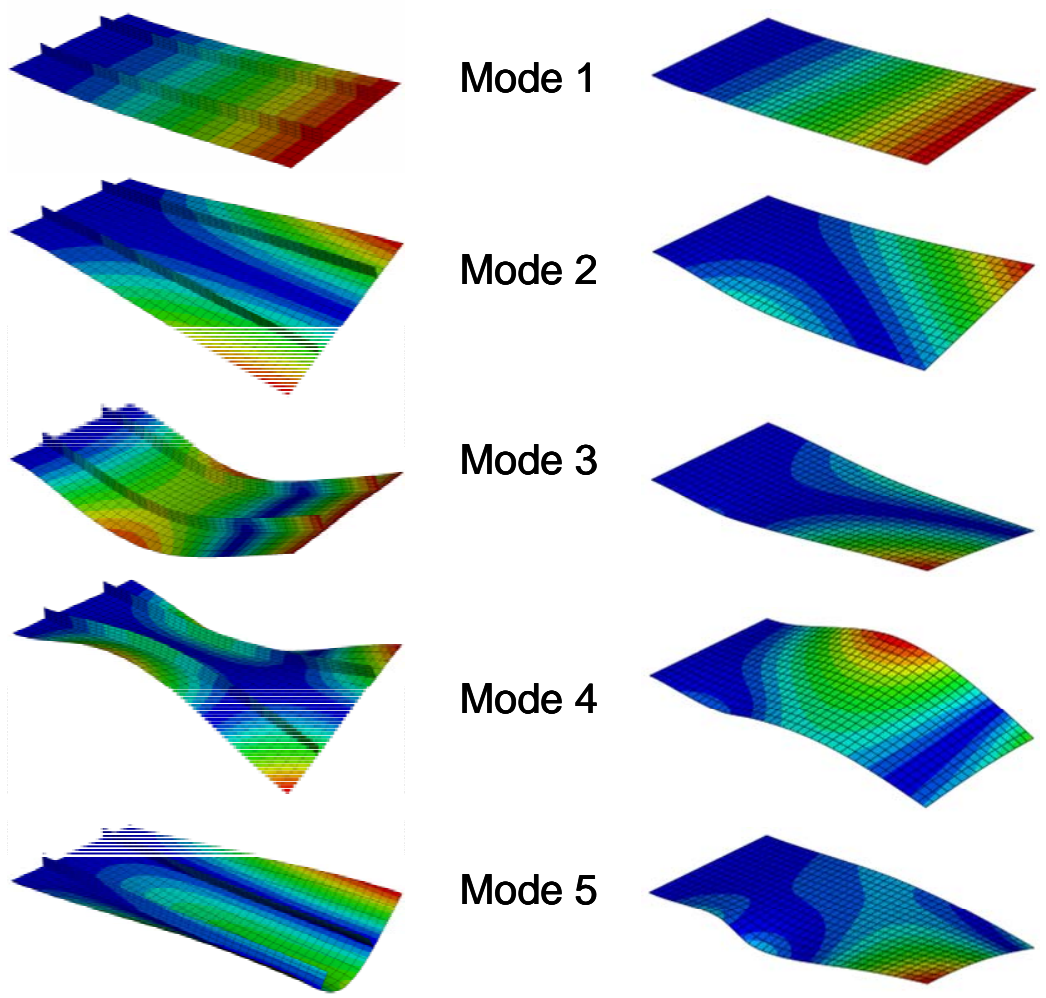

Figure 6. Stiffened-panel without damage: comparison of mode shapes for a geometric-scale factor 0.5

\section{Model 2: Stiffened-Panel with Circular Damage:}

In this model, the stiffened panel shown in Figure 1 is modified by introducing damage in the form of a 2.5 inch circular cut-out at the center. The dimensions, material properties, and loading are shown in figure 7 . The stiffeners are cut at locations in the middle as shown schematically the figure. The damage size in the stiffened-panel and the equivalent plate are kept the same. Figures 8 and 9 illustrate the finite element models for the reference case and for the equivalent plate.

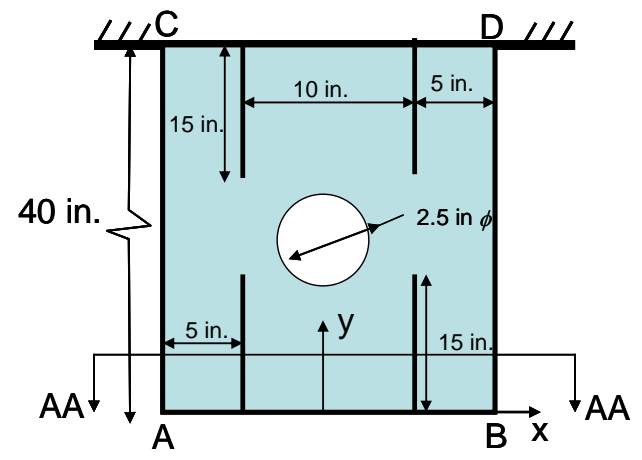

$$
\begin{array}{ll}
\text { Material: } & \\
\text { Young's Modulus } & =0.1 \text { E08 psi } \\
\text { Poisson's Ratio } & =0.3 \\
\text { Density } & =0.0975 \mathrm{lbm} / \mathrm{in}^{3}
\end{array}
$$
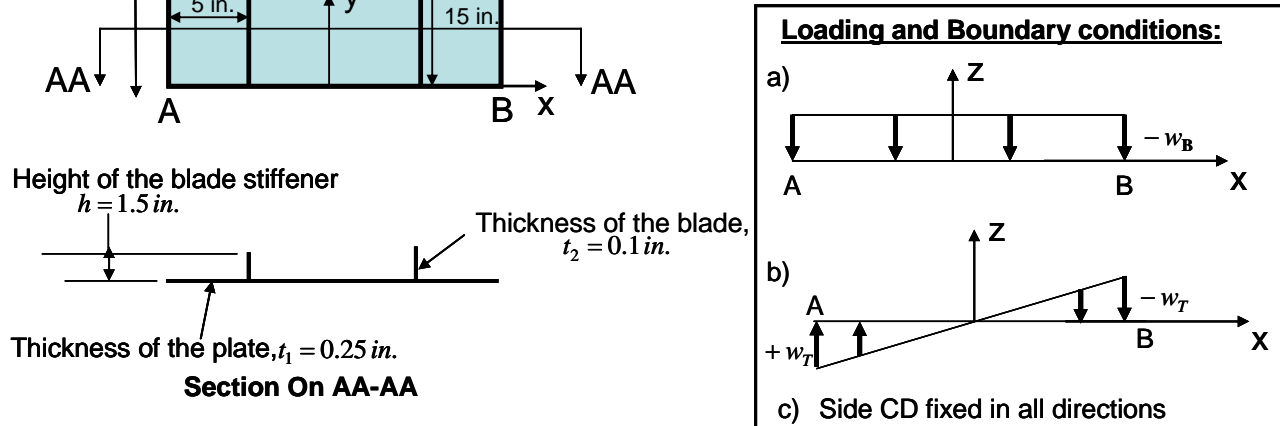

Figure 7. Geometry of the stiffened panel with circular damage used in the equivalent plate generation 


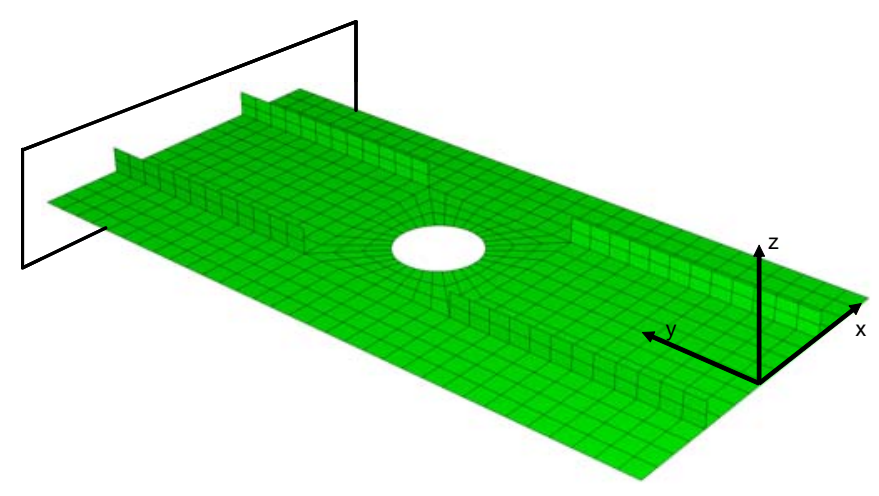

Figure 8. Finite element model of the reference stiffened panel with circular damage

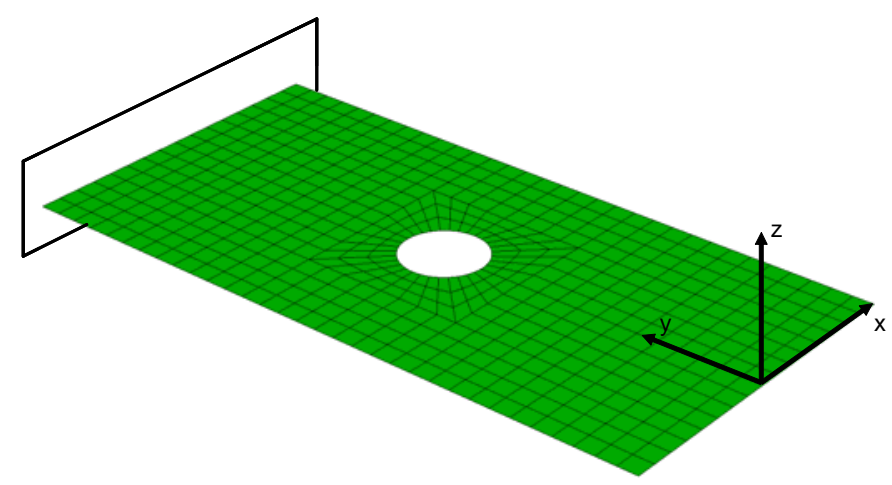

Figure 9. Finite element model of the equivalent plate with circular damage

Linear variation of the thickness is assumed along the length of the plate. The thickness distribution obtained for scale factor 1.0 is presented in reference 8 . Similar results proportional to the geometrical scaling were obtained for scale factor 0.5 and 0.2 and are not reported here.

For this model, the results were presented in reference 8 for geometric-scale factor 1.0. Hence, the deformation of the scaled equivalent plate is compared with the reference and stiffened panels for geometric-scale factor 0.5 and 0.2 in Figure 10. As in the previous case, the deformation of the model is in good agreement with the reference solution. The two simple examples presented here clearly demonstrate the ability of the method to reproduce the static response of the structures using the geometric-scale factors defined in the present study.

Next, the frequency response of the scaled equivalent plate is compared with the reference solution in Figure 11. The error is within 1.5 percent in all the cases. This example demonstrates the selection of geometric-scaling factors along with the frequency-scaling factors that are able to reproduce the frequency response accurately.

The range of frequency-scale factors used in the study is compared in Table 2 for various geometric-scale factors along with the point masses. The frequency-scale factors are varied from 0.7 to 2.0 as the geometric-scale is decreased from 1.0 to 0.2 . In this case, the total mass added to the scaled equivalent plate to match the frequencies is not as high as was noticed in the case of the stiffened-panel without damage. Here also, no attempt was made to optimize the point mass locations. 


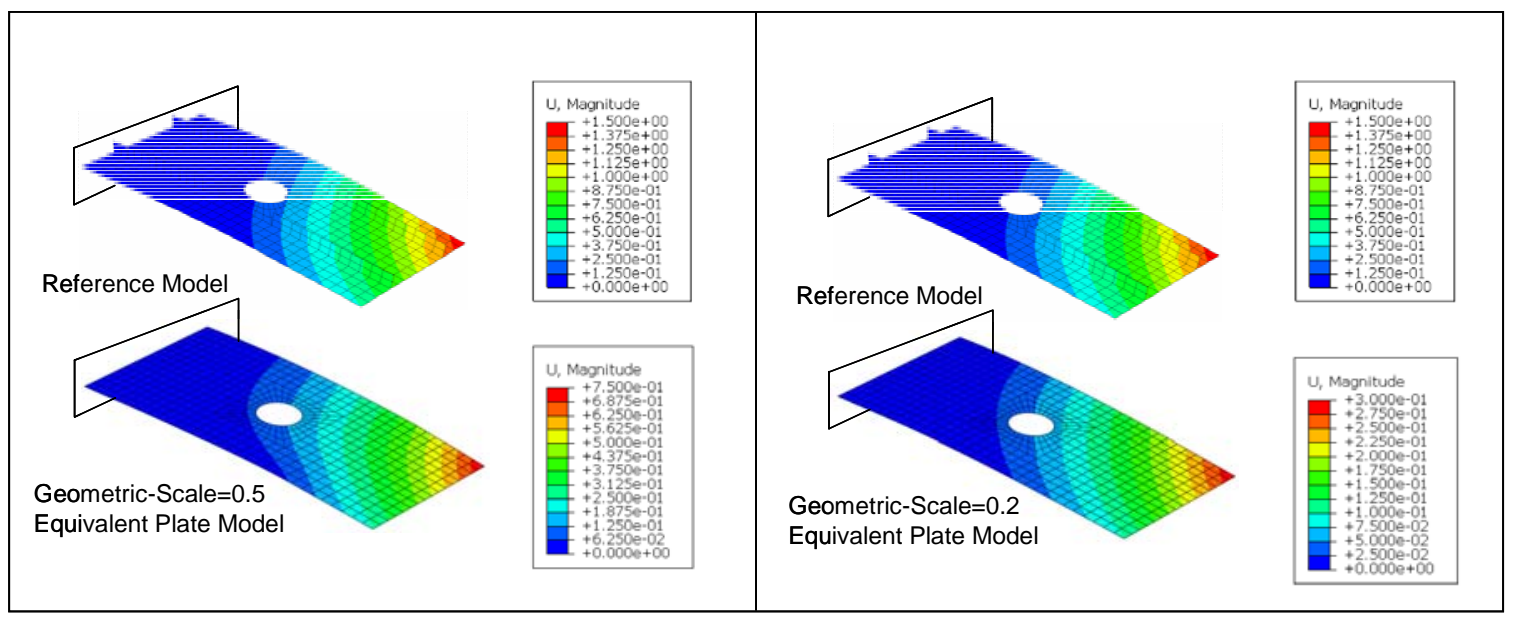

Figure 10. Stiffened-panel with circular damage: deformation comparison-geometric scale-factors 0.2 and 0.5 .
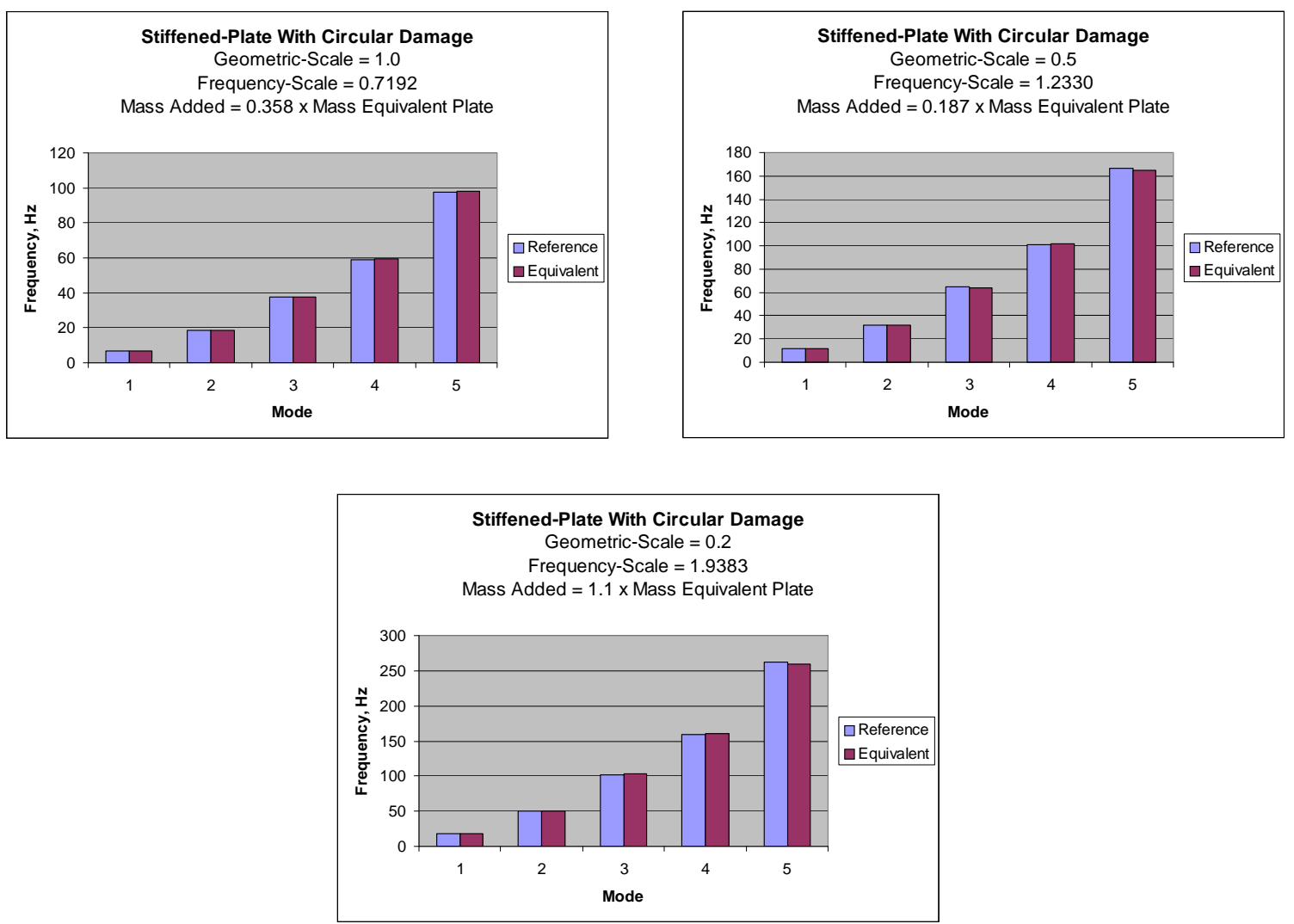

Figure 11. Stiffened-panel with circular damage: Frequencies comparison for geometric scale=1.0, 0.5 and 0.2 
Table 2. Stiffened-panel with circular damage: frequency-scale factors and added mass values

\begin{tabular}{|c|c|c|}
\hline Geometric-Scale & Frequency-Scale & Mass Added \\
\hline 1.0 & 0.7192 & $0.358 \times$ Mass Equivalent Plate \\
\hline 0.5 & 1.2330 & $0.187 \times$ Mass Equivalent Plate \\
\hline 0.2 & 1.9383 & $1.100 \times$ Mass Equivalent Plate \\
\hline
\end{tabular}

In this case, the mode shapes for all the geometric-scale factors look almost same; hence, the mode shapes are compared for geometric-scale factor of 0.2 in Figure 12. Also, the lower mode shapes agree better than the higher mode shapes.

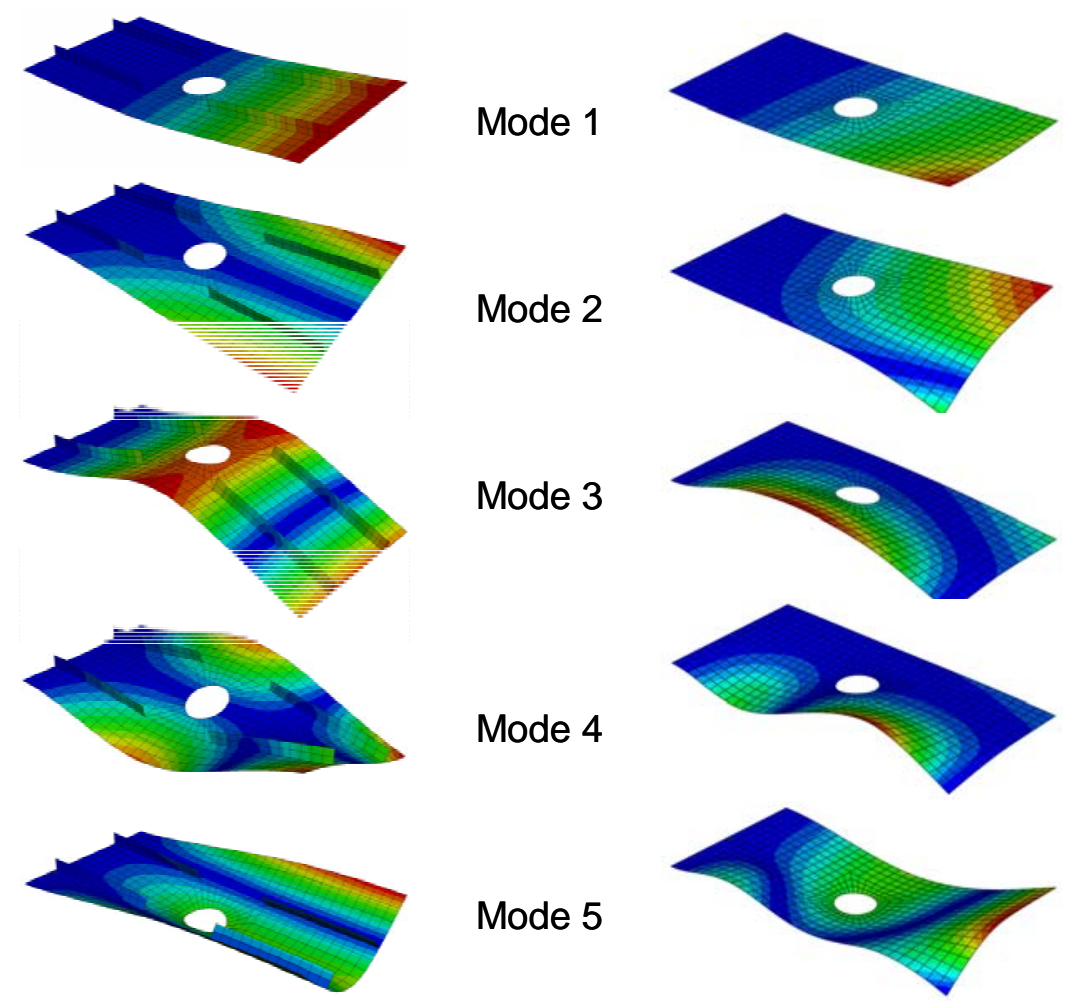

Figure 12. Stiffened-panel with circular damage: mode shape comparison- geometric-scale factor 0.2

\section{Numerical Example: Typical aircraft wing structure}

Next, the methodology employing geometrical-scale factors combined with frequency-scale factors to predict the static and dynamic response is demonstrated in a typical aircraft wing structure. A detailed finite element model of the typical wing structure used in the demonstration is shown in Figure 13. The model has two spars and 26 ribs. The major dimensions of the wing are also shown in Figure 13. An equivalent plate model was created by extracting the elements of the upper skin and zeroing out the vertical coordinates of all nodes (not shown here). First, equivalent plate geometries were generated by matching the stiffness of the wing structure for geometric-scale factors 1.0 and 0.2. Next, the generated scaled equivalent plates were used to match frequencies. 


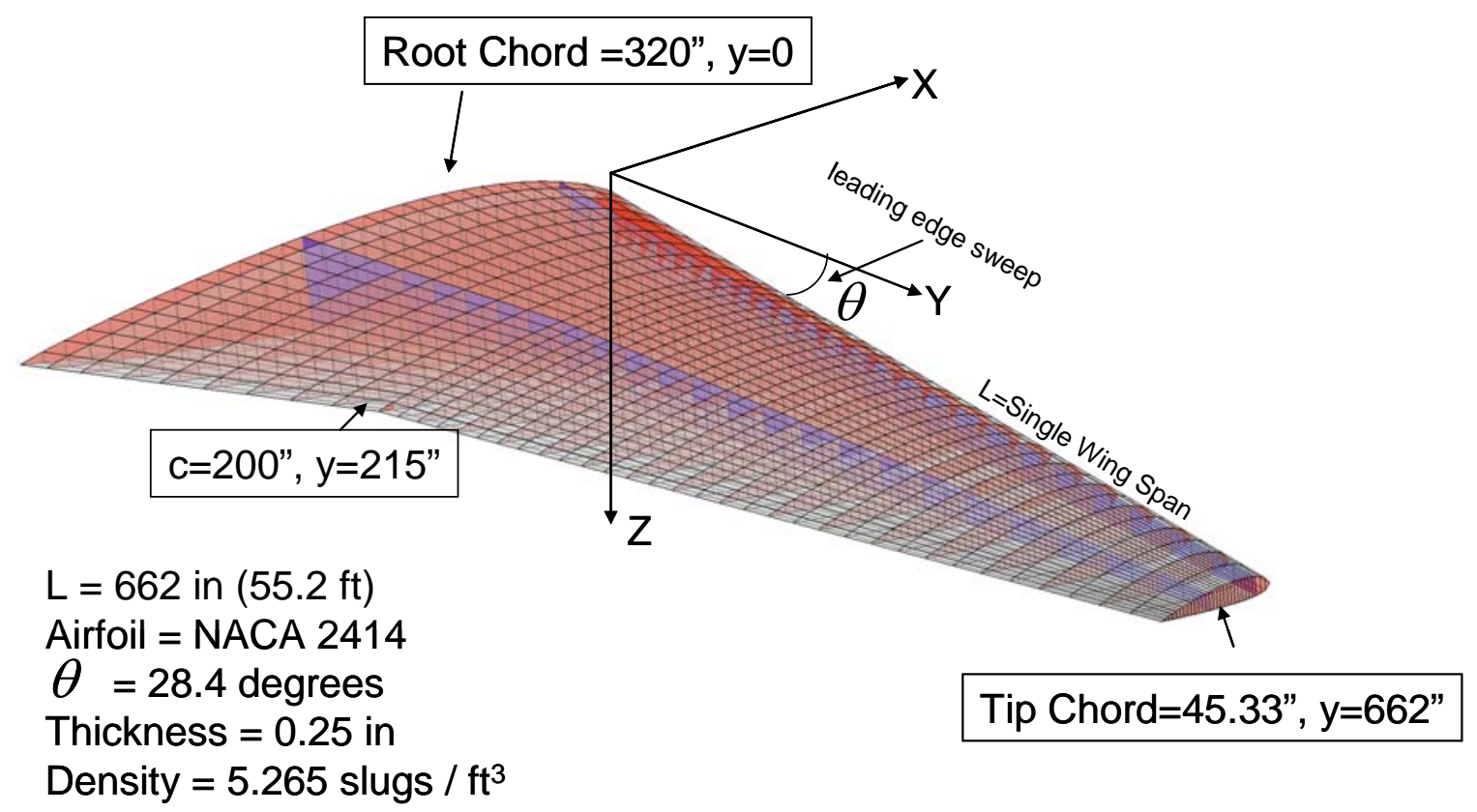

Figure 13. Typical aircraft wing: finite element model

A linear polynomial along the width direction and three piecewise linear polynomials in the length direction are used to characterize the thickness distribution. The thickness values are not reported here. The deformation obtained from the scaled equivalent plate model is compared with the reference aircraft wing deformation in Figure 14 for geometrical-scale factors 1.0 and 0.2 . The deformation from the equivalent plate is in very good agreement with the deformation from the reference wing structure. This example along with the two simple examples in the previous section clearly demonstrated the ability of the optimization procedure presented here to generate scaled equivalent plate models that can predict the static response accurately.

Next, the equivalent plate models developed for the geometric-scale factor 1.0 and 0.2 were used to predict the frequency response of the aircraft wing structure using the procedure developed in Section III. The frequencies obtained using the scaled equivalent plates are compared with the reference wing structure in Figure 15. Most of the errors are within 2 percent. For mode 5 , the error is about 5 percent (for geometric-scale factor 1.0).

The frequency-scale factors and the total point mass added to the equivalent plate are given in Table 3 . The frequency-scale factor varies from 0.15 to 0.45 as the geometric-scale factor varied from 1.0 to 0.2 . The total mass added in the case of geometric-scale factor 0.2 is about 15 times the mass of the equivalent plate. However, no attempt is made to optimize the number of point mass locations. There is a need to develop methods to minimize the total mass added to the equivalent plate.

Finally, the mode shapes are compared in Figure 16 for the case of geometric-scale factor 0.2. Similar shapes were obtained for the scale factor 1.0. Lower mode shapes agree better than the higher mode shapes. 


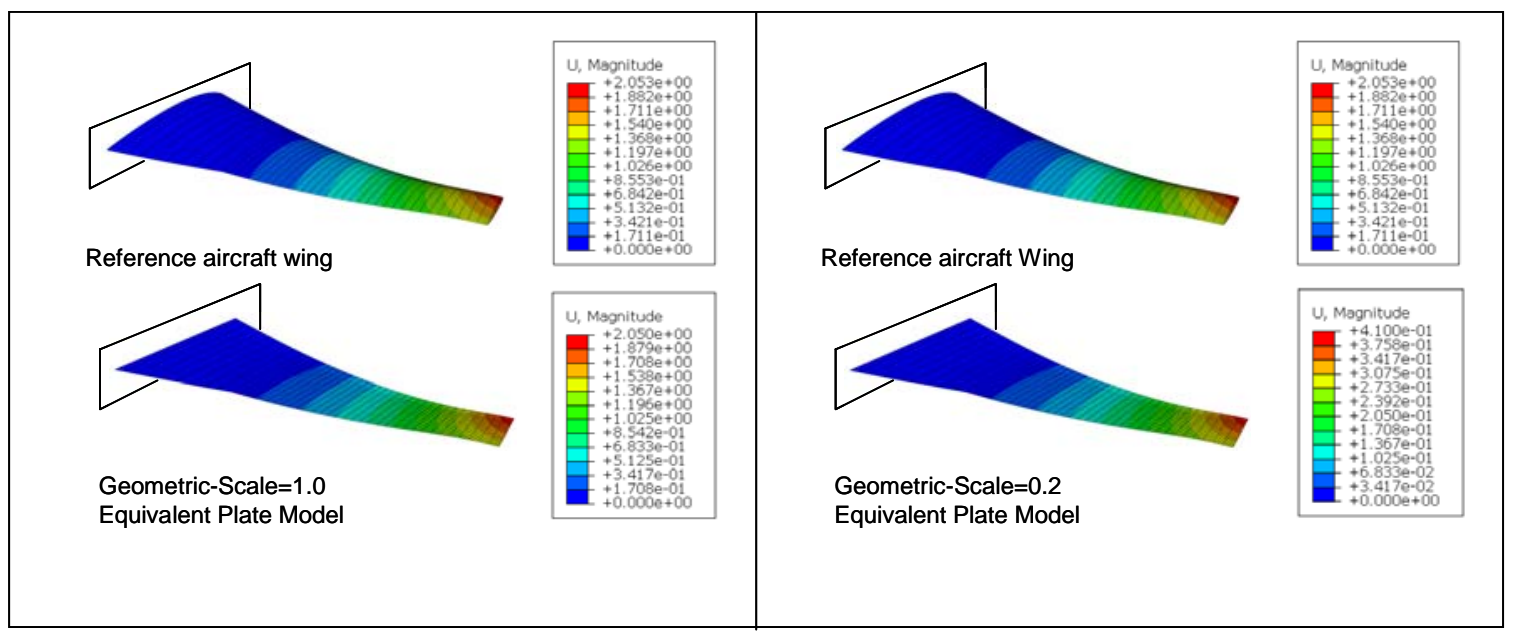

Figure 14. Aircraft wing structure: deformation comparison for geometric-scale factors 1.0 and 0.2
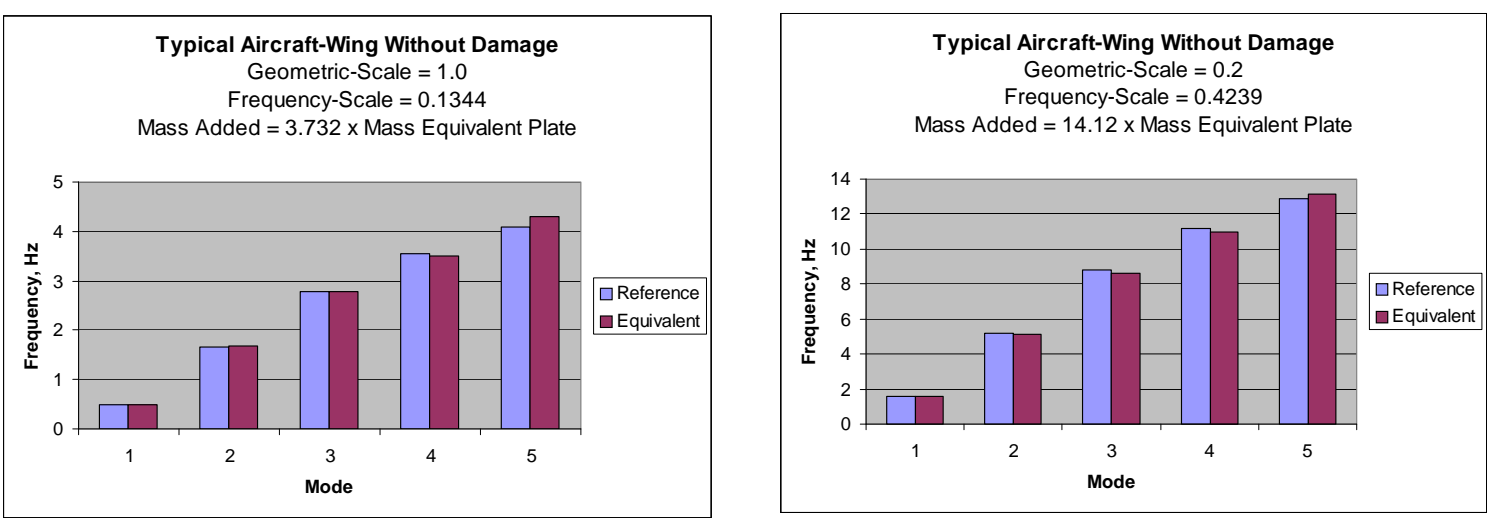

Figure 15. Aircraft wing structure: frequencies comparison for geometric-scale factors 1.0 and 0.2

Table 3. Aircraft wing structure: frequency-scale factors and total point mass added

\begin{tabular}{|c|c|c|}
\hline Geometric-Scale & Frequency-Scale & Mass Added \\
\hline 1.0 & 0.1344 & $3.732 \times$ Mass Equivalent Plate \\
\hline 0.2 & 0.4239 & $14.120 \times$ Mass Equivalent Plate \\
\hline
\end{tabular}



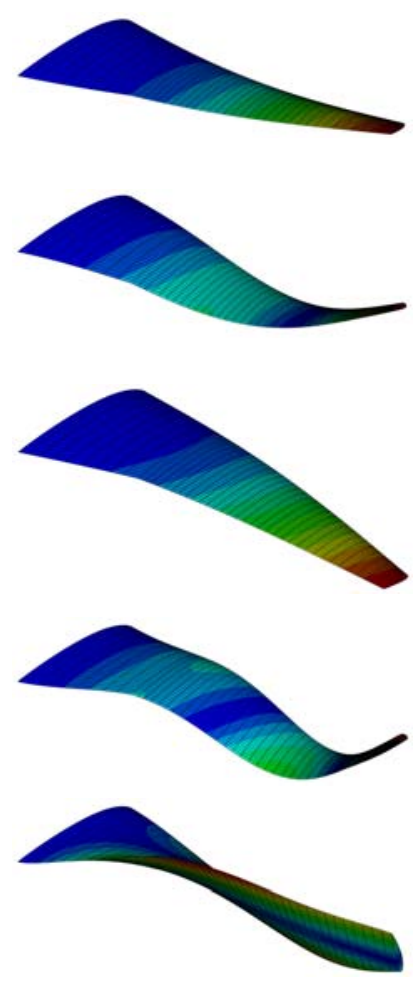

\section{Mode 4}

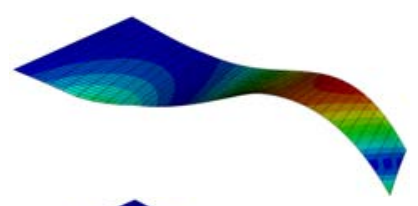

Mode 5

Figure 16. Aircraft wing structure: mode shape comparison for geometric-scale factor 0.2

\section{Concluding Remarks}

A process to generate an equivalent plate based on an optimization approach to predict the static and dynamic response of flight vehicle wing structures was proposed. Geometric-scale factors and frequency-scale factors are defined to construct an equivalent plate with any desired scale to use in simulation and wind tunnel experiments. The geometric-scale factor is used to scale stiffness, load, and displacements of the equivalent plate from the flight vehicle. It is shown that the stiffness and the displacements of the equivalent plate are scaled linearly with the geometric-scale factor, whereas the load is scaled with the square of the geometric-scale factor. The scaled stiffness of the reference flight vehicle is matched first to construct the geometry of the equivalent plate. Next scaled flight vehicle frequencies are matched by adding arbitrary point masses along the length of the equivalent plate.

Two simple stiffened-plate examples, one with damage and another without damage, were used to demonstrate the accuracy of the optimization procedure proposed. Geometric-scale factors ranging from 0.2 to 1.0 were used in the analyses. In both the examples, the static and dynamic responses of the reference stiffened-panel solution are matched accurately. The scaled equivalent plate predicted the first five frequencies of the stiffened-panel within two percent.

Finally, the proposed equivalent plate procedure was demonstrated in a more realistic typical aircraft wing structure. Two scale equivalent plate models were generated using the geometric-scale factors 1.0 and 0.2. Both equivalent plate models predicted the static response of the wing structure accurately. The equivalent plate models reproduced the first five frequencies of the wing structure within five percent.

Mode shape fidelity was not a requirement for the equivalent plates, but their mode shapes were examined. Lower modes matched better than higher modes. 


\section{References}

1. Giles, G. L. "Equivalent Plate Modeling for Conceptual Design of Aircraft Wing Structures," AIAA1995-3945, Presented at $1^{\text {st }}$ AIAA Aircraft Engineering, Technology and Operations Congress, Los Angeles, CA, Sept. 19-21, 1995.

2. Mason, B. H., Stroud, W., Krishnamurthy, T., Spain, C., and Naser, A., "Probabilistic Design of a Wind Tunnel Model to Match the Response of a Full-Scale Aircraft," AIAA-2005-2185, Presented at 46th AIAA/ASME/ASCE/AHS/ASC Structures, Structural Dynamics and Materials Conference 13th AIAA/ASME/AHS Adaptive Structures Conference, Austin, Texas, Apr. 18-21, 2005.

3. Giles, G. L. "Equivalent Plate Analysis of Aircraft Wing Box Structures with General Planform Geometry,” Journal of Aircraft, Vol. 23. No. 11, pp. 858-864, 1986.

4. Giles, G. L. "Further Generalization of Equivalent Plate Representation for Aircraft Structural Analysis," Journal of Aircraft, Vol. 26. No. 1, pp. 67-74, 1989.

5. Stone, S. C., Henderson, J. L., Nazari, M. M., Boyd, W. N., Becker, B. T., Bhatia, K. G., Giles, G. L. and Wrenn, G. A., "Evaluation of Equivalent Plate Solution (ELAPS) in HSST Sizing," AIAA-2000-1452, Presented at 41st AIAA/ASME/ASCE/AHS/ASC Structures, Structural Dynamics, and Materials Conference and Exhibit, 41st, Atlanta, GA, Apr. 3-6, 2000.

6. Mavris, D. N., and Hayden, W. T., "Probabilistic Analysis of an HSCT Modeled with an Equivalent Laminated Plate Wing,” AIAA-1997-5571, Presented at AIAA and SAE, 1997 World Aviation Congress, Anaheim, CA, Oct. 13-16, 1997.

7. Krishnamurthy, T. and Mason, B. H., "Equivalent Plate Analysis of Aircraft Wing with Discrete Source Damage”, AIAA-2006-2218, Presented at 47th AIAA/ASME/ASCE/AHS/ASC Structures, Structural Dynamics, and Materials Conference, 14th AIAA/ASME/AHS Adaptive Structures Conference, Newport, Rhode Island, May 1-4, 2006.

8. Krishnamurthy, T., and Eldred, L.B., "Frequency Response of an Aircraft Wing with Discrete Source Damage Using Equivalent Plate Analysis, ” AIAA-2007-2144, Presented at 48th AIAA/ASME/ASCE/AHS/ASC Structures, Structural Dynamics, and Materials Conference, Honolulu, Hawaii, Apr. 23-26, 2007.

9. Roskam, J., Holgate, T., and Shimizu, G, "Elastic wind-tunnel Models for Predicting Longitudinal Stability Derivatives of Elastic Airplanes,” Journal of Aircraft, Vol. 5, No. 6, pp. 543-550, 1968.

10. Heeg J., Spain, C. V., and Rivera, J. A., "Wind Tunnel to Atmospheric Mapping for Static Aeroelastic Scaling,” AIAA-2004-2044, Presented at 45th AIAA/ASME/ASCE/AHS/ASC Structures, Structural Dynamics, and Materials Conference, Palm Springs, California, Apr. 19-22, 2004.

11. Anonymous, Getting Started with ABAQUS, Version 6.5, ABAQUS, Inc., Providence, RI 02909.

12. Anonymous, DOT, Design Optimization Tools, User's Manual, Version 5.0, Vanderplaats Research \& Development, Inc., Colorado Springs, CO, 80906. 